\title{
Study on the Interaction between Upper and Lower Parts of Super high-rise Tube
}

\author{
Songan Liu*, Yafei Liu \\ School of Resource Exploration \& Civil Engineering, The Engineering \& Technology College of \\ Chengdu University of Technology, Leshan, Sichuan 614000, China
}

Email: Isa_lyf@sina.com

Keywords: Tube in Tube Structure, Upper and Lower Structure, Finite Element, Static Action

\begin{abstract}
Study on analysis of interaction between the upper and lower is a hot research topic at present. It is produced with the rapid development of high rise buildings and computer technology. At present, super structure and foundation are paid more attention as engineering problem. In the design of building structure, the structure of the upper column as a separate structure is fixed on the basis of this traditional method without considering the influence of interaction between superstructure and foundation and foundation. The results were different from the actual, especially in high rise buildings. The unreasonable phenomenon is more and more obvious. Use the response spectrum method analyze of the horizontal structure of seismic dynamic. Compare and summarize the different strengthening layer setting scheme, structure displacement, internal force of the earthquake response, the following related conclusion: strengthening layer can effectively reduce the horizontal displacement of the structure, and with the increased number of layer reduction will increase. The dynamic response analysis under the earthquake provides some reference and suggestions for the seismic design of this kind of structure.
\end{abstract}

\section{Introduction}

The design concept of interaction between structure and foundation has gained much attention in academic and engineering fields [1-3]. Its calculation theory has made a great progress, especially in the numerical analysis theory. The finite element, infinite element analysis method and random combination etc. analysis method greatly improve the seismic action calculation theory. Finite element analysis for seismic response of structure and foundation interaction has been widely used, the geometric properties and mechanical properties of finite element can effectively simulate the structure and foundation of medium [4,5].

Infinite element is a solution to the infinite foundation of numerical method developed in the 1970s. When designing the structure or hydraulic structure, the structure and the foundation are considered as two independent systems. Generally, the foundation as a fixed bearing of the upper structure to solve the internal force of super structure under load. Only consider the interaction between the foundation and the basics without considering the interaction of structure and foundation. In the earthquake, the response of the structure is different from the ground [6-9]. The movement of structures under seismic action is the result of the interaction of foundation and structure. In the process of seismic design, to meet the safety of structures, if only designed according to the conventional design method, which may cause an accident. There is no shortage of experience and lessons at home and abroad in engineering. Therefore, study on the dynamic response of structure and foundation interaction, seeking a reasonable analysis method, truthfully reflect the actual situation of structure and foundation interaction, has far-reaching theoretical significance and engineering significance. 


\section{Theoretical analysis of upper and lower structures}

\subsection{Mechanism of interaction between upper and lower structures}

With the interaction of theory and practice of in-depth understanding, mechanism of high-rise building structure and foundation interaction becomes more and more profound. The upper structure, the foundation and the basics are interrelated and influence each other.

\subsubsection{Influence of superstructure stiffness}

The stiffness of the structure is comprehensive of horizontal stiffness, vertical stiffness and bending stiffness. Generally speaking, with the increase of layers, stiffness and bending stiffness is raised rapidly in the first few layers, then quickly slowed, tend to a stable value at last. While the vertical stiffness increases with the number of the floor increase to a certain law, reaches a certain level, but also stable at final, which is different from the first two, more layers [10,11]. Whether the plane frame structure, frame structure filling wall, or shear wall structure, their stiffness also increase as so. When the foundation, foundation and constant load, deflection of the upper structure stiffness increases is the basis, the internal force is reduced, but the superstructure increases, the upper and lower structure is to increase their stress at the expense of the internal force decreases the foundation.

\subsubsection{Influence of foundation stiffness}

In the upper structure rigidity and foundation conditions, with the internal force of foundation stiffness increases, the relative immunity decreased [12]. On the contrary, the upper structure of the secondary stress with the foundation stiffness decreases significantly increased. Because the settlement difference (i.e. relative immunity) increase, will inevitably lead to greater times of the stress in the superstructure. From the view of reducing the stress of the foundation,we should reduce the foundation stiffness. Therefore, the structural type should be taken into account in the basic scheme. If the superstructure is a flexible structure, as long as meet certain requirements, foundation should not be rigid $[13,14]$. On the contrary, for the high compression frame structure of foundation, because it is sensitive to differential settlement, foundation should not be soft.

\subsection{Finite element method}

In the field of science and technology, many mechanical problems and physical problems can be attributed to the solution of the control equations under fixed boundary conditions (ordinary differential equations or partial differential equations) problem. But be able to obtain the exact solution is a relatively simple equation by using an analytical method, geometry rather rules. For most of problems the engineering and technology, due to some nonlinear characteristic equation or the complex of shape, you will be unable to get an analytic answer. To solve this kind of problem is usually two ways. Introducing simplifying assumptions, equations and geometric boundary will be simplified as able to handle the situation in order to get the solution in the reduced state the problem. But this method only in limited circumstances is feasible, because too much simplification may cause a large error and wrong solution answer. Another way is to keep the problem of complexity, using the numerical approximate and numerical calculation method for the minimization problem, with the rapid development of electronic computer and widely used, has been gradually tending to adopt this method to solve the complex engineering problem. Finite element method is a novel and effective numerical method to solve these complicated engineering problems.

\subsection{Substructure method for structural analysis}

In analysis of large-scale structure with finite element method, the number of total stiffness matrix elements often exceed the allowable computer storage capacity. In addition, for the system composed by several structural forms, such as high-rise buildings - frame shear wall system, often caused the ill-conditioned equation by the difference of structure stiffness characteristics. Because of the above reasons, must adopt some method of structure segmentation, to make the number of 
elements in the total stiffness of the segmented structure be suitable for the storage capacity of the computer or let the equation does not appear ill conditioned. Structural analysis of the substructure method was originally developed for solving finite element problems of large and complex aircraft structure, and then used for analysis of substructure method of interaction.

\subsubsection{The basic idea of substructure method}

The basic idea of the substructure method is a structure be divided into several non-overlapping substructures and according to the needs of the substructure be divided into several levels. Further the sub structure is divided into internal and external degrees of freedom too. External freedom is connected with the upper sub structure of the degrees of freedom. As Fig. 1 substructure shown, substructure 1 and substructure 2 are designated as the primary structure (first level). Substructure 3 is the sub structure of second sub structure. Draw $X$ as the external nodes of them, all the nodes and sub structure 3 the degree of freedom is composed of main structure (top level structure) degrees of freedom.

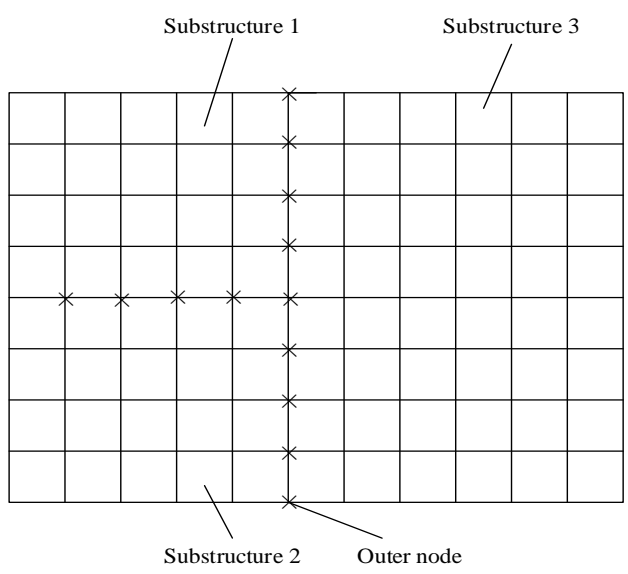

Figure 1. Sub structure partition

\subsubsection{The structural stiffness matrix and the load vector}

A segment as shown in Fig. 2, sub structure from the total architecture, the nodes can be divided into two types: one is internal node is not connected with other sub structures (represented by i), the other is a boundary node connected with other sub structures (represented by b).

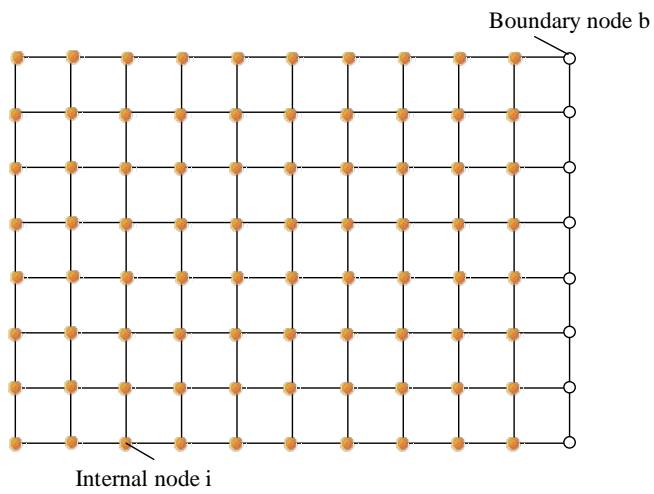

Figure 2 . Sub structure partition 


\section{Static analysis and dynamic analysis considering the interaction of upper}

\section{1 computational model}

In this paper, in order to facilitate comparison, draw meaningful conclusions, this paper uses two kinds of ANSYS based on the finite element model. Including the foundation and without foundation model. The following were introduced for the two models. This project for the postal telecommunications network management center building, the modeling process of the upper structure in strict accordance with the construction plans. I model of interaction model in Figure 3 (a); II model does not consider the interaction model, see 3 (b)

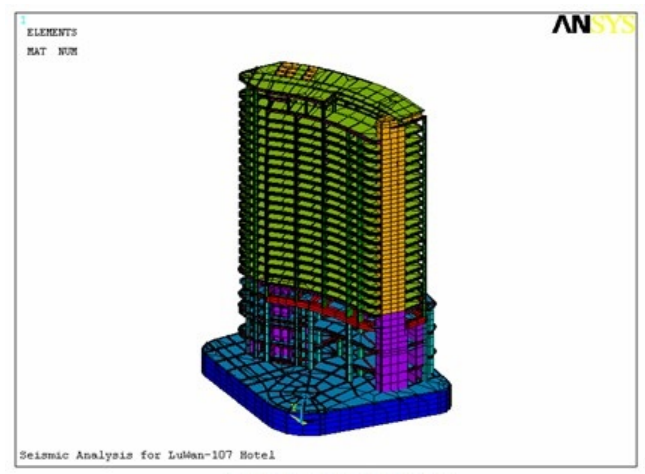

(a) Model I

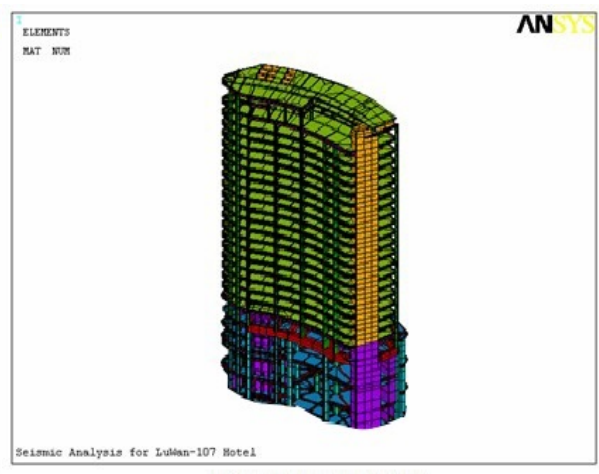

(b) Model II

Figure 3. Model graph

Model I uses equivalent complex model, the SOLID45 element is used to discrete, see3 (a) model. The overall number of degrees of freedom is 117010 . The total number of nodes is 32878. A total of 33434 units, of which the upper structure beam column with beam element, the inner cylinder wall, floor, frame the corner with the shell element, a total of 19003 units and the lower foundation unit number 14431 raft by SHELL63 unit, unit number is 347. The ground is replaced by a large area in the half space, the SOLID45 unit, a total of 14084 units

Model II does not consider the total effect through the model, the superstructure is fixed at the bottom of the independent structure. It is the traditional computing model commonly used, see3(b). The structure of the degrees of freedom is 73914, the total number of nodes is 16121 units, a total of 19003 units (BEAM4) beam column element number 2289, number (BEAM4) 1172, floor unit (SHELL63) number 11812, the shear wall element (SHELL63) number 3730 total tonnage of 95796.4 tons of superstructure.

\subsubsection{Internal force results of upper and lower structural members}

We use 1-4 component represent for the shear wall corner, 5-26 member for four corner posts, 27-30 shear wall around two layer underground. The calculation results such as shown in table 1:

Table 1. Axial force comparison of each layer

\begin{tabular}{c|c|c|c|c|c|c|c|c}
\hline \multirow{2}{*}{ floor } & \multicolumn{2}{|c|}{ column 6 } & \multicolumn{2}{c|}{ column10 } & \multicolumn{2}{c}{ column16 } & \multicolumn{2}{c}{ column21 } \\
\cline { 2 - 9 } & model I & model II & model I & model II & model I & model II & model I & model II \\
\hline 1 & 11332.4 & 11330.0 & 11276.2 & 11368.2 & 11297.9 & 11289.3 & 11324.8 & 11338.7 \\
\hline 7 & 10211.3 & 10233.4 & 10197.8 & 10193.2 & 102443.7 & 10234.9 & 10186.8 & 10223.5 \\
\hline 14 & 7322.4 & 7328.7 & 8425.6 & 8538.9 & 7478.9 & 7439.8 & 8325.9 & 8437.0 \\
\hline 21 & 4998.1 & 4978.0 & 5243.1 & 5497.5 & 5329.7 & 5497.1 & 5502.1 & 5389.4 \\
\hline 28 & 2783.4 & 2648.9 & 2342.3 & 2134.1 & 2013.4 & 2178.2 & 2341.5 & 2381.4 \\
\hline percent & \multicolumn{3}{|c|}{0.87} & \multicolumn{3}{c|}{1.18} & \multicolumn{3}{c}{-1.97} & \multicolumn{3}{c}{1.14} \\
\hline
\end{tabular}


It can be seen from table 1. After considering the interaction, the mean axial force increases generally. The change value of $-1.97 \%$. As the basis had a basin shaped settlement, the settlement of the middle side is greater than the edge of the upper structure, to participate in the development of the resistance in the settlement of side column due to relative extrusion and loading, and unloading in column due to relatively stretching. If changing the distribution of internal force of the upper structure, the components of the upper part of the structure is not safe. Agree with column loading and unloading algebra are zeros. The pile foundation can effectively reduce the uneven settlement, and between inner and outer plane stiffness using only small span unbonded prestressed plate connection. In this case, the proportion inside the change was not obvious. The change of the superstructure with a variety of factors, in some cases, the side column internal force increase is larger in the design can not be ignored.

Table 2. The ratio of inner and outer cylinders in each layer of the superstructure

\begin{tabular}{c|c|c|c|c}
\hline \multirow{2}{*}{ floor } & \multicolumn{2}{|c|}{ model I } & \multicolumn{2}{c}{ model II } \\
\cline { 2 - 5 } & The outer cylinder & The inner cylinder & The outer cylinder & The inner cylinder \\
\hline 1 & $53.14 \%$ & $46.86 \%$ & $50.62 \%$ & $49.38 \%$ \\
\hline 7 & $52.12 \%$ & $47.88 \%$ & $50.51 \%$ & $49.49 \%$ \\
\hline 14 & $51.03 \%$ & $48.97 \%$ & $50.32 \%$ & $49.68 \%$ \\
\hline 21 & $49.32 \%$ & $50.68 \%$ & $48.43 \%$ & $51.57 \%$ \\
\hline 28 & $47.38 \%$ & $52.62 \%$ & $45.36 \%$ & $54.64 \%$ \\
\hline
\end{tabular}

Share from table 2 inside and outside the tube load ratio data can be seen, with the increase of the construction layer, the outer cylinder ratio decreased, the inner cylinder ratio gradually increased. Up to 28 layers, the inner cylinder ratio reached 52.56\%. The interaction of outer cylinder share more loads, the outer cylinder is slightly larger than the ratio of the maximum difference of grief about $0.6 \%$. This is because the outer cylinder was a result of the foundation settlement of the crown under increasing load.

\section{Summary}

This article through the analysis the interaction of superstructure internal force under gravity loads and settlement rules, and does not consider interaction through compare the calculation results obtained the following conclusions: consider a common role, superstructure component internal gravity redistribution phenomenon. Since foundation basin shaped settlement, the settlement of the middle side over the edge of the upper structure involved in the resistance development of this settlement, the side column due to relative compression loading. If changing the distribution of internal force of the upper structure, the components of the upper part of the structure is not to cover the whole. Reflect the settlement law is basically consistent with the value calculated from the finite element and the measured value. The settlement of the middle and peripheral minor settlement, a basin shaped distribution. Considering the interaction of the finite element calculation results can better reflect the actual law. Based on the design, pay more attention to it. The lower part of the structure involved in the interaction of nurses, to the upper structure, foundation and foundation of the analysis may lead to large errors.

\section{References}

[1] Yuan Z J. Finite element analysis of super trussed tube structure[J]. Journal of Harbin University of Civil Engineering \& Architecture, 2000.

[2] Bouzidi R, Buytet S, Van A L. A numerical and experimental study of the quasi-static deployment of membrane tubes[J]. International Journal of Solids \& Structures, 2013, 50(5):651-661. 
[3] Luo L C, Chen M. Motorcycle frame structure based on finite element modeling of static strength analysis and improved design[J]. Machinery, 2012.

[4] Rathnaweera G, Dong R, Hajj M, et al. Performance of aluminium/Terocore ${ }^{\circledR}$; hybrid structures in quasi-static three-point bending: Experimental and finite element analysis study[J]. Materials \& Design (1980-2015), 2014, 54:880-892.

[5] Martin K A, Ehsani M R, Bjorhoude R. Field testing of monotube span type sign structures[J]. 1985.

[6] Mansol M M, Ismail N I, Mahamad Basri M H, et al. Analysis of thin walled tube Al 3003 H12 under quasi-static axial crush mode using finite element method[J]. 2014.

[7] Cheng X H, Ma F. Beam Frame Supported Shear Wall Structure Based on the ANSYS Finite Element Static Analysis of Beam-Type Transfer Floor[J]. Advanced Materials Research, 2013, 788:508-510.

[8] Kunihiro N. EXHAUST STATIC TUBE SUPPORT STRUCTURE AND INTERNAL COMBUSTION ENGINE EQUIPPED WITH SAME:, WO/2015/182414[P]. 2015.

[9] Wang Q, Papageorgiou D T. Using electric fields to induce patterning in leaky dielectric fluids in a rod-annular geometry[J]. Ima Journal of Applied Mathematics, 2016:hxw017.

[10] Weinstein B, Ponslet E. BSC Downtube Structure Mechanical Design and Analysis[J].

[11] Chen M, Xing D, Zhao Y, et al. Experimental research and finite element analysis on joints of Sun Valley steel structure for the Expo Axis project[J]. Journal of Building Structures, 2010, 31(5):34-41.

[12] Saur J, Neubauer F M, Strobel D F, et al. Three-dimensional plasma simulation of Io's interaction with the Io plasma torus: Asymmetric plasma flow[J]. Journal of Geophysical Research Space Physics, 1999, 104(A11):25105-25126.

[13] Lindsay J D, Hermans M A, Hada F S. Leakage control system for treatment of moving webs: US, US 6280573 B1[P]. 2001.

[14] Goldsmith A N. Remote control system: US, US2345472[P]. 1944. 